\title{
Plasma cholesterol level after hepatopancreatobiliary surgery provides information on the postoperative clinical course
}

\author{
Gennaro Nuzzo • Ivo Giovannini
}

Received: 26 May 2010/ Accepted: 25 August 2010/Published online: 7 September 2010

(C) Springer-Verlag 2010

Hypocholesterolemia is commonly considered an index of malnutrition. Conversely, major surgical procedures such as liver resection or pancreaticoduodenectomy and the possibly related adverse events such as sepsis, major bleeding, or other complications may cause a reduction in plasma cholesterol to values even lower than $30 \mathrm{mg} / \mathrm{dl}$. Therefore, in this case, the severity of hypocholesterolemia often becomes an index of severity of illness with relevant prognostic value [1-3]. In spite of the fact that cholesterol may be influenced by various other factors, the evolution of hypocholesterolemia (the trend toward worsening or resolution, or the protracted persistence of severe hypocholesterolemia) provides useful information on the clinical evolution of the patient.

It is worth outlining the mechanisms which are involved in the development of hypocholesterolemia and some practical cues for its interpretation. Plasma cholesterol behaves like albumin, whose levels tend to decrease in conditions of stress, such as after surgical trauma and with the occurrence of complications (sepsis, liver insufficiency, and hemorrhage). The mechanisms involved in decreasing cholesterol may include the effect of inflammatory mediators, the insufficient hepatic synthesis of cholesterol, and hemodilution, the latter being related to blood loss and fluid reinfusion [4]. The final effect is cumulative, and the severity of hypocholesterolemia reflects the importance of the adverse factors which determine the severity of illness. In our experience, after liver resection, the reduction in cholesterol, with respect to the preoperative value, is

G. Nuzzo $(\bowtie) \cdot$ I. Giovannini

Hepatobiliary Surgery Unit, Department of Surgical Sciences,

Catholic University School of Medicine,

Largo Agostino Gemelli 8, 00168 Rome, Italy

e-mail: gennaro.nuzzo@rm.unicatt.it cumulatively related to the magnitude of the operation, the possible presence of sepsis and liver insufficiency, and the decrease in hematocrit; in this case, a persistently severe fall in cholesterol correlates with poor prognosis [3, 5] (Fig. 1). The tendency for a greater fall in cholesterol after major abdominal surgery such as after pancreaticoduodenectomy, compared to what is observed after other operations, may at least partly be explained by the magnitude of the operation.

The assessment of postoperative cholesterol should also take into account its relative value as a fraction (postoperative/preoperative ratio) or as a percentage of the preoperative level. In any case, the protracted persistence of cholesterol below $60-50 \mathrm{mg} / \mathrm{dl}(\approx 1.5-1.3 \mathrm{mmol} / \mathrm{l})$ is often associated with severe sepsis, at times occult sepsis which, if not recognized and rapidly treated, may become lethal $[3,5]$. On the contrary, a transient decrease in cholesterol is less important as long as the levels subsequently steadily increase with the resolution of the underlying problem. Therefore, although plasma cholesterol cannot be used as a precise diagnostic tool, the changes along time may provide useful prognostic information.

Cholestasis, if simultaneously present, exerts an opposite influence on the behavior of plasma cholesterol. In fact, cholestasis increases cholesterol, and levels even greater than $400 \mathrm{mg} / \mathrm{dl}$ may be observed in nonsurgical patients developing obstructive jaundice without a previous history of dyslipidemia. The underlying mechanisms are the reduced biliary excretion of cholesterol and the release by the liver into the blood of the cholesterol-rich lipoprotein-X. In any case, in critically ill and postoperative patients, cholestasis moderates the degree of hypocholesterolemia which is related to other adverse factors (major extent of surgery, sepsis, liver insufficiency, major bleeding, or other complications). This occurs with or without complete 


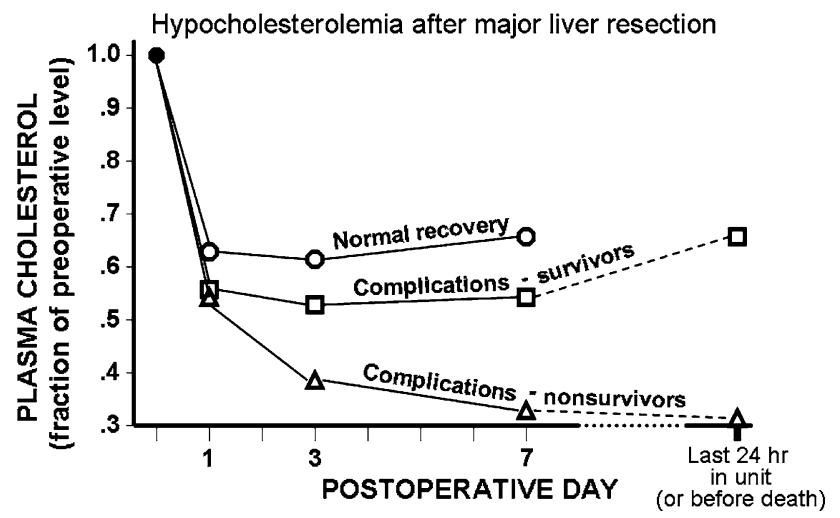

Fig. 1 Postoperative evolution of plasma cholesterol concentration in patients with normal recovery, or with complications (mainly sepsis) followed by recovery or death. See text. Reproduced in simplified form with permission from Clinical Chemistry (2003) 49:317-319 [5]. ${ }^{\circ} 2003$ AACC

biliary obstruction, and the increase in cholesterol correlates better with the increase in the enzymes of cholestasis than with the level of bilirubin. Therefore, in cholestasis, the prognostic role of cholesterol is hampered. Postoperatively, however, especially when surgery involves relief of the obstruction, cholesterol decreases and regains a prognostic role. An estimation of the effect of cholestasis may be obtained by considering that cholesterol increases by approximately $7 \mathrm{mg} / \mathrm{dl}(\approx 0.2 \mathrm{mmol} / \mathrm{l})$ per $100 \mathrm{U} / \mathrm{l}$ increase in alkaline phosphatase (n.v. 98-279 U/l) [1, 2, 4]. These notions also explain why patients with extreme illness and multiple organ failure may not show very severe hypocholesterolemia if cholestasis is simultaneously present.

In summary, the following concepts should be highlighted: (1) the factors which may prevent the occurrence of very severe hypocholesterolemia are a high basal cholesterol and/or the presence of cholestasis; (2) severe hypocholesterolemia has worse prognostic implications in the presence of cholestasis; and (3) the trend toward resolution of severe hypocholesterolemia has good prognostic value, except when this is caused by cholestasis.

Very severe but transient hypocholesterolemia is not necessarily relevant. As already mentioned, it is severe and persistent hypocholesterolemia that is often associated with poor prognosis, while the resolution of hypocholesterolemia tends to be associated with recovery. Individual variations and the multifactoriality of hypocholesterolemia do not allow the definition of precise landmarks of risk. However, in a published study [5], no patient with sepsis and organ failure after liver resection survived, among those who remained for more than 6 days with cholesterol below $58 \mathrm{mg} / \mathrm{dl}(1.5 \mathrm{mmol} / \mathrm{l})$, or below $40 \%$ of the preoperative level (Fig. 1).

Perhaps, hypocholesterolemia does not simply passively reflect severity of illness, but may also contribute actively to poor prognosis $[4,5]$. In fact, hypocholesterolemia might reflect: (1) a reduction of the lipoproteins which protect against microbial products and toxic mediators; (2) a reduced antioxidant capacity of blood through various mechanisms, including the fact that vitamin $\mathrm{E}$ (antioxidant) is transported by lipoproteins; and (3) a reduced availability of cholesterol in conditions of increased requirement. Indeed, inadequate availability of cholesterol may limit the synthesis of stress hormones (cortisol) and the production of new cells which take part in host defense and tissue repair; it is sufficient to remember that pus is one of the richest materials in cholesterol. Finally, cholesterol is essential for the synthesis of many other cells, including those involved in liver regeneration after partial hepatectomy. An additionally relevant aspect is that the endogenous production of cholesterol is a very demanding metabolic process. Although it may appear counterintuitive, fat emulsions for parenteral nutrition contain only an irrelevant amount of cholesterol.

In surgical trauma and sepsis, plasma triglycerides tend to follow a pattern which is similar to that of cholesterol; however, in sepsis with severe hypocholesterolemia, the development of a dissociated pattern with the simultaneous occurrence of hypertriglyceridemia may reflect an even worse degree of illness, with amplification of the inflammatory response and imminent risk of death [4]. The main practical implication, apart from the interruption or the reduction in rate of fat infusion, is that this peculiar pattern signals an extreme severity of illness, in which the only hope of survival is often related to the rapid and aggressive eradication of sepsis [4]. Therefore, there is a prognostic role of hypertriglyceridemia which potentiates that of hypocholesterolemia. This type of hypertriglyceridemia develops in the absence of fat infusion (fat infusion can worsen it), and its nature is as yet undefined. It may reflect enhanced release of triglyceride-rich lipoproteins into blood, perhaps as an extreme attempt at defense, impaired triglyceride removal from blood, or both.

While it may appear a paradox, nutritional state has very little to do with the hypocholesterolemia which is observed in sepsis and after surgical trauma. The mechanisms by which the infusion of nutritional substrates and in particular of fat can modify plasma cholesterol depend on the release of cholesterol from cell membranes in exchange with other components of the emulsions (which contain only trace amounts of cholesterol). Cholesterol may also increase with the infusion of amino acids, perhaps because these provide cholesterol precursors and/or facilitate the synthesis of the apoprotein components of lipoproteins. Experimental attempts to treat hypocholesterolemia as such include the infusion of fat emulsions with protective properties against endotoxin and the infusion of cholesterol; however, the effects are not yet well determined [4]. 
In any case, nutritional support has a fundamental role in the treatment of critically ill patients because it provides basic substrate also for cholesterol synthesis in spite of the modest impact on plasma cholesterol level. Apart from the setting of sepsis and other surgical critical illnesses, hypocholesterolemia is observed in other situations, which include congenital conditions, malnutrition, hyperthyroidism, chronic inflammatory diseases, several types of cancer and myeloproliferative diseases, some patients on renal replacement treatment and elderly patients. Hypocholesterolemia is generally considered a marker of pathophysiologic frailty, and in preoperative surgical patients it may involve a greater predisposition to postoperative complications. With regard to this issue, important preventive measures include the treatment of underlying diseases (for instance unrecognized infections) and the improvement of nutritional state in malnourished patients.

Recapitulating the main concepts, soon after surgery cholesterol decreases as a component of the acute phase response; thenceforth, it tends to return toward the preoperative value as shown in Fig. 1. With the occurrence of sepsis cholesterol remains low; additional causes of hypocholesterolemia are liver insufficiency and hemorrhage, but these causes are more obviously recognizable. Cholesterol remains low also when sepsis is not associated with surgery. Hypocholesterolemia does not specifically anticipate a diagnosis of sepsis, but reflects the abnormal persistence of an acute phase response. For example, in a postoperative patient who is not fully recovering, although without clear evidence of complications, hypocholesterolemia suggests the presence of occult sepsis and should promote adequate investigations to confirm or rule it out. Following the surgical treatment of sepsis, cholesterol further decreases because of the surgical trauma; thereafter, a gradual reversal of hypocholesterolemia represents a helpful tool, in the difficult assessment of this type of patients, to evaluate progressive recovery. Some inter- patient variability and cholestasis, if present, can modify the described patterns.

In conclusion, in the presence of septic complications after major surgical procedures such as liver resection or pancreaticoduodenectomy, the level of plasma cholesterol and triglycerides may provide useful information on the clinical evolution of the patient. In the future, a better understanding of the processes involved will certainly improve the treatment of critically ill patients. Presently, the most concrete clinical implication of severe hypocholesterolemia, associated or not with hypertriglyceridemia, is the need for the rapid resolution of the underlying process, which is often represented by postoperative abdominal sepsis or other causes of sepsis.

Conflict of interest None.

\section{References}

1. Nuzzo G, Giovannini I, Boldrini G, Chiarla C, Giuliante F, Cavicchioni C, Marrocco M (1994) Changes of plasma lipid profiles in postoperative and septic patients. In: Mutz NJ, Koller W, Benzer H (eds) Proceedings of the 7th European congress on intensive care medicine, Innsbruck. Monduzzi, Bologna, pp 1059-1062

2. Giovannini I, Boldrini G, Chiarla C, Giuliante F, Vellone M, Nuzzo G (1999) Pathophysiologic correlates of hypocholesterolemia in critically ill surgical patients. Intensive Care Med $25: 748-751$

3. Nuzzo G, Giovannini I, Giuliante F, Ardito F, Chiarla C (2009) Sepsis after liver resection: predisposition, clinical relevance and synergism with liver dysfunction. In: Dionigi R (ed) Recent advances in liver surgery. Landes Bioscience, Austin, pp 245-259

4. Chiarla C, Giovannini I, Giuliante F, Zadak Z, Vellone M, Ardito F, Clemente G, Murazio M, Nuzzo G (2010) Severe hypocholesterolemia in surgical patients, sepsis, and critical illness. J Crit Care 25:361.e7-361.e12

5. Giovannini I, Chiarla C, Greco F, Boldrini G, Nuzzo G (2003) Characterization of biochemical and clinical correlates of hypocholesterolemia after hepatectomy. Clin Chem 49:317-319 